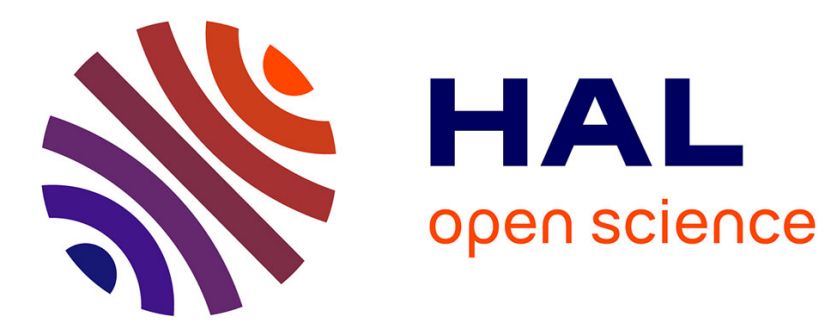

\title{
Entre État et marché. Une nouvelle régulation sanitaire pour les pays en développement?
}

Stéphane Tizio

\section{To cite this version:}

Stéphane Tizio. Entre État et marché. Une nouvelle régulation sanitaire pour les pays en développement?. Revue Tiers Monde, 2004, 179, pp.643-663. 10.3406/tiers.2004.5513 . hal-02502820

\section{HAL Id: hal-02502820 \\ https://u-bourgogne.hal.science/hal-02502820}

Submitted on 9 Mar 2020

HAL is a multi-disciplinary open access archive for the deposit and dissemination of scientific research documents, whether they are published or not. The documents may come from teaching and research institutions in France or abroad, or from public or private research centers.
L'archive ouverte pluridisciplinaire HAL, est destinée au dépôt et à la diffusion de documents scientifiques de niveau recherche, publiés ou non, émanant des établissements d'enseignement et de recherche français ou étrangers, des laboratoires publics ou privés. 


\title{
Entre État et marché. Une nouvelle régulation sanitaire pour les
}

\section{pays en développement?}

\section{Stéphane Tizio}

\begin{abstract}
Stéphane Tizio - Between State and market : A new sanitary regulation for developing countries ?

This paper is positioned in a critical perspective of the most recent international recommendations in matters of health in developing countries and particularly in Sub- Saharan African countries. A brief history of health policies shows that the promotion of these new instruments is greatly owed to the limits of the State and market models of organising health systems. Proceeding from the neo-institutional models of such new health policies, we shall demonstrate the implications and limits consisting, among other things, of discarding the political and ethical dimensions of regulating health systems.
\end{abstract}

\section{Citer ce document / Cite this document :}

Tizio Stéphane. Entre État et marché. Une nouvelle régulation sanitaire pour les pays en développement ?. In: Tiers-Monde, tome 45, n¹79, 2004. Varia. Démocratie et développement, pauvreté au Liban, emploi domestique en Afrique du Sud, boom du soja au Brésil... pp. 643-663;

doi : https://doi.org/10.3406/tiers.2004.5513

https://www.persee.fr/doc/tiers_1293-8882_2004_num_45_179_5513

Fichier pdf généré le 30/03/2018 


\title{
ENTRE ÉTAT ET MARCHÉ. UNE NOUVELLE RÉGULATION SANITAIRE POUR LES PAYS EN DÉVELOPPEMENT?
}

\author{
par Stéphane Tızıo*
}

Ce papier s'inscrit dans une perspective critique des recommandations les plus récentes des organisations internationales en matière de santé dans les pays en développement et particulièrement dans les pays d'Afrique subsaharienne. Un bref historique des politiques sanitaires subsahariennes montre que la promotion de ces nouveaux instruments est en grande partie issue de l'épuisement des modèles étatique et marchand d'organisation des systèmes de santé. Puis, à partir des fondements néoinstitutionnels de ces nouvelles politiques sanitaires, nous en montrons les implications et les limites, qui consistent, entre autres, à évacuer les dimensions de l'éthique ou du politique de la régulation des systèmes de santé.

Depuis un certain nombre d'années, les politiques sanitaires mises en œuvre ou recommandées tant par les gouvernements locaux que par les organisations internationales (OMS ou Banque mondiale) se réclament plus ou moins explicitement des approches néo-institutionnelles et, plus particulièrement, de la théorie des coûts de transaction (Williamson, 1985) qui fonde la «bonne gouvernance " des systèmes sanitaires dans les pays en développement (World Bank, 1998). Ces nouvelles recommandations ont pour objet de favoriser des "approches contractuelles " des systèmes de santé (Carrin et al., 1997 ; Perrot, 1999) ou encore des "stratégies sectorielles de santé " (World Bank, ibid.). Elles visent à réintroduire un État régulateur des systèmes de santé, troisième voie entre la libéralisation totale et l'étatisation

\footnotetext{
* Maître de conférences à l'Université de Bourgogne, GRFS/LEG (UMR CNRS n 5118), Pôle d'économie et de gestion.

Revue Tiers Monde, t. XLV, n" 179, juillet-septembre 2004
} 
outrancière, deux options qui, si elles ont suscité beaucoup d'espoirs dans le passé, ont été sources de grandes désillusions.

À cet égard, le cas de l'Afrique subsaharienne est tout à fait éclairant ; il est possible de distinguer, dans l'historique des politiques de santé subsahariennes, trois époques (Tizio, Flori, 1997 ; Tizio, 1999) : dans un premier temps, les politiques sanitaires subsahariennes marquées par la déclaration d'Alma-Ata (1978) visent à atteindre un objectif égalitariste de "santé pour tous", instrumentalisé dans les faits par le développement géographique de l'offre sanitaire de base et le financement quasi exclusivement public des dépenses de santé. Dans un second temps, à partir de 1987, l'Initiative de Bamako fait évoluer les politiques de santé vers un désengagement assez marqué des États : sur le plan organisationnel, les systèmes de santé décentralisés doivent être autogérés par des associations d'usagers ; sur le plan financier, les mêmes usagers doivent alors payer pour les soins qu'ils reçoivent. La troisième époque des politiques de santé subsahariennes - la période actuelle - voit réapparaître l'État dans les politiques de santé nationales, comme simple partenaire et comme l'instance qui fixe les " règles du jeu» des accords contractuels entre les différents partenaires - publics ou privés - des systèmes sanitaires.

Dans un premier temps, à la lumière d'un bref historique des politiques de santé subsahariennes, nous mettons en perspective la logique qui sous-tend le recours aux accords structurels dans les recommandations récentes de la Banque mondiale et de l'Organisation mondiale de la santé : une réflexion sur le rôle et la nature de l'État met en lumière, à travers ces évolutions, la nécessité de redéfinir le cadre et les moyens de son intervention dans les politiques sanitaires subsahariennes. L'analyse des implications et des limites de l'économie néoinstitutionnaliste nous permettra de montrer, dans un second temps, que la recherche d'un nouveau paradigme de développement sanitaire, fondé sur le partenariat entre les différents acteurs des systèmes de santé, aurait avantage à intégrer, en plus des dimensions strictement contractuelles, des dimensions politique et éthique.

LES TROIS ÉPOQUES

DE LA RÉGULATION SANITAIRE SUBSAHARIENNE

1. De la "santé pour tous" à la "maladie pour chacun"

La déclaration d'Alma-Ata, ratifiée en 1978 par les États membres de l'oms, vise à officialiser la stratégie de "santé pour tous en 
l'an 2000 "; les États membres y sont invités à proposer et à promouvoir des plans d'action nationaux et régionaux afin d'atteindre cet objectif. Dès l'article premier, la conférence affirme que la santé est un droit fondamental de l'être humain et que les inégalités en termes de santé entre les peuples doivent être éliminées. Cela implique ainsi simultanément une égalité d'accès aux soins et une égalité de traitement pour un même besoin. Aussi le système de soins doit-il être organisé de sorte qu'il n'existe plus d'obstacle pour l'acquisition des soins, c'est-à-dire que ces derniers soient identiquement disponibles pour l'individu, quel que soit son revenu. En termes de politique sanitaire, cela suppose que l'offre soit adéquatement répartie, au regard de la répartition géographique des besoins repérés par la participation active des communautés au niveau local - participation communautaire -, mais également que soit menée une politique de gratuité ou de subvention des soins de santé primaires pour les consommateurs, cela afin d'éliminer l'inégal accès aux soins, en particulier entre la campagne et les villes, et d'atténuer l'impact du montant des revenus sur cet accès.

La logique égalitariste qui fonde les politiques sanitaires à AlmaAta est sous-tendue par une conception tutélaire du rôle de l'État. Cette conception, introduite en théorie par les travaux de Musgrave (1959), conduit à justifier les interventions de l'autorité publique dans des domaines variés pour des raisons qui tiennent à la préservation ou à la garantie de l'intérêt général, c'est-à-dire finalement à la poursuite d'un objectif égalitariste. L'autorité publique doit alors mettre en œuvre tout ce qui est possible pour limiter les iniquités, mais également pour identifier et repérer la nature des besoins de la population qu'elle a en charge. C'est donc sur l'autorité publique - et sur elle seule - que repose tant la charge de la diffusion de l'offre sanitaire dans les régions les plus enclavées que la subvention des soins. Ainsi, dans le cadre de la déclaration d'Alma-Ata, l'État finance et organise le système de santé dans son ensemble.

Cependant, dans le contexte économique qui se dégrade à la suite des chocs pétroliers, la mise en ouvre des soins de santé primaires sur le modèle d'Alma-Ata est stoppée ; la diffusion géographique de l'offre de santé ne peut se poursuivre, faute de moyens pour la mener à bien. Ainsi, l'obstacle géographique à l'égalité d'accès aux soins perdure puisque l'arrêt du développement des structures prévues exclut de facto une partie de la population du système de santé. Par ailleurs, ni l'égalité en termes d'accès aux soins, tenue en échec par la baisse du financement public - l'infrastructure locale n'est pas développée : les prix en termes de distance ne sont alors pas égaux pour tous -, ni la prise en charge du financement des soins, via la solvabilisation de la 
demande, ne sont possibles'. Dans ce contexte, l'échec des réformes étatistes des systèmes de santé traduit l'effondrement du pouvoir des autorités publiques en matière de régulation de l'activité économique en général et, en particulier, des systèmes de santé. Cela conduit les gouvernements locaux, mais surtout les organisations internationales, à revitaliser l'organisation et le financement des systèmes de santé subsahariens en lançant l'Initiative de Bamako en 1987.

L'Initiative de Bamako comprend deux volets : d'une part, il s'agit de mobiliser des ressources privées qui, via la tarification des soins, doivent se substituer au financement public défaillant; d'autre part, il s'agit d'intégrer le recouvrement des coûts dans le principe de la participation communautaire, qui devient "financement communautaire " (Parker, Knippenberg, 1991).

Dans le cadre du volet financier de la réforme, l'amélioration de la qualité est conçue comme un objectif prioritaire. La stagnation de la demande de soins de santé biomédicaux, offerts par les centres de santé publics, est analysée comme devant résulter du choix des usagers qui préfèrent les prestataires informels et/ou traditionnels. L'affectation des ressources collectées à l'amélioration de la qualité de la médecine officielle doit permettre de mettre celle-ci à nouveau en concurrence avec les tradipraticiens et les médecins clandestins. À partir de l'impulsion donnée par l'aide internationale ${ }^{2}$, la fréquentation des centres de santé doit alors augmenter.

Sur le plan organisationnel, la participation communautaire, qui existait déjà, est complétée par le volet financier de l'initiative de Bamako : le recouvrement des coûts. Les prérogatives des communautés sont étendues, elles doivent repérer les indigents - ceux qui ne peuvent payer les soins -, mais également s'occuper de leur prise en charge sanitaire.

En première analyse, la participation communautaire, qui consiste à décentraliser la gestion du sanitaire vers les collectivités locales, peut se voir attribuer les vertus égalitaristes qui président à l'élaboration des stratégies de soins de santé primaire; mais la tarification des soins qui lui est associée est intrinsèquement inégalitaire : au sein d'une communauté, la population se départage entre les individus qui peuvent payer les soins et ceux qui en sont dans l'impossibilité. Afin de répondre à ce problème et en l'absence de toute possibilité de gratuité

1. Cette solvabilisation passe par la pratique de politiques de gratuité et/ou de subvention des soins, politiques qui deviennent intenables, avec la baisse des budgets sanitaires et sociaux.

2. L'aide internationale est nécessaire aux projets de recouvrement des coûts, au moins dans leur phase de démarrage, puisque la qualité des soins, censée faire augmenter la fréquentation des postes de santé, ne peut se mettre en place spontanément, sans ressources au départ. 
intégrale des soins de santé, la poursuite d'un objectif d'équité passe par la pratique de la discrimination positive entre les deux catégories de populations : ceux qui ne peuvent payer les soins seront pris en charge gratuitement ou à un tarif plus faible que ceux qui peuvent faire face financièrement au prix des interventions de santé. La réalisation d'une telle équité est subordonnée à l'efficacité financière du système de tarification dans la mesure où la prise en charge des indigents doit être financée par les ressources mobilisées auprès des individus qui paient : la santé financière des services de santé communautaire est capitale, surtout dans le cas où les autorités centrales n'interviendraient pas, faute de moyens.

Cependant, d'importants dysfonctionnements subsistent. De faibles taux de couverture des coûts et la persistance d'une demande pour les soins de santé officiels sont loin de faire exception (Nolan, Turbat, 1995). Les problèmes préexistant à l'adoption de la réforme restent et ils plaident en faveur de la reconsidération du rôle de l'autorité publique en matière d'organisation et de financement du secteur de la santé.

\section{La période actuelle: genèse des " approches contractuelles 》}

Jusque dans les années 1980, la tendance en Afrique était à l'augmentation de l'intervention de l'État dans l'économie, notamment dans les domaines sociaux, afin de pallier le manque d'initiative privée (Jacquemot, 1988). Cependant, depuis le début de la décennie 1980, le désengagement de l'État est au centre des réflexions et des initiatives politiques et économiques des organisations internationales (Hugon, 1991). Un processus de désétatisation se répand qui fait passer les sociétés d'Afrique subsaharienne de la "surconsommation d'État " à "l'État mou ", avec la faillite des institutions et l'essor d'une économie clandestine. La mise en place d'un système de santé viable pour les pays d'Afrique subsaharienne demande de reconsidérer le rôle que peut jouer l'autorité publique dans l'organisation et le financement de la santé. Ni État omniprésent (Alma-Ata), ni État minimal (Initiative de Bamako), les politiques de santé actuelles se fondent sur un État partenaire des autres acteurs du système de santé : c'est la genèse des "approches contractuelles" des politiques de santé en Afrique subsaharienne.

Jusqu'à la conférence d'Alma-Ata, les systèmes de santé subsahariens sont marqués par le peu de diversité des acteurs ; dans la mesure où la plupart des systèmes de santé sont intégralement étatisés, on ne 
distingue pas finalement le producteur direct des services du financeur, puisque celui qui délivre les services est placé sous la tutelle de l'autorité publique. Néanmoins, depuis la mise en œuvre des politiques sanitaires s'appuyant sur la déclaration d'Alma-Ata, les communautés deviennent des partenaires non négligeables au sein des systèmes sanitaires, qui s'intercalent entre la tutelle étatique et le producteur de soins proprement $\mathrm{dit}^{1}$. À ces communautés viennent s'ajouter des organisations non gouvernementales qui vont prendre une part de plus en plus active, non seulement dans la production directe des services, mais également dans la définition même des politiques sanitaires au niveau local. Au sein des systèmes de santé, coexistent des producteurs privés, comme des cliniques ou des médecins libéraux, avec un secteur traditionnel. Enfin, depuis quelques années, des organisations de financement collectif, indépendantes des pouvoirs publics, apparaissent, telles des assurances ou des mutuelles, ou encore des entreprises qui, outre l'aspect strictement financier du système, peuvent parfois participer à la production des services.

La pluralité des acteurs des systèmes montre la disparition graduelle de la dichotomie hermétique entre secteur public et secteur privé, et pointe la nécessité de permettre à ces différents acteurs de coordonner leurs actions, de manière à fournir des réponses efficaces aux problèmes de santé des populations. Tel est l'objet de l'approche contractuelle.

L'évolution des systèmes de santé est marquée simultanément par la diversification des acteurs et la séparation des rôles. Ainsi la structure d'un système de santé se définit-elle par les interactions des acteurs et des rôles qu'ils jouent : la multiplicité des combinaisons possibles montre alors l'hybridation des formes de coordination entre acteurs des systèmes de santé ; celles-ci sont alors définies par des contrats passés entre les différentes entités composant le système sanitaire. Ces contrats peuvent lier deux ou plusieurs acteurs du système et portent tant sur l'ensemble du système que sur des objectifs particuliers, par exemple la promotion de la santé en milieu scolaire ou la lutte contre certaines pathologies fortement prévalentes. En particulier, lorsque la poursuite d'un objectif d'équité est au cœur du dispositif, un contrat peut réunir à la fois les populations, les prestataires de santé et un - ou plusieurs - financeurs. La participation communautaire, pierre angulaire des réformes des systèmes de santé depuis les années 1970, n'échappe pas à la contractualisation. L'implication des

1. Lorsque les systèmes ne sont pas intégralement étatiques, un secteur privé - à but lucratif ou non - peut exister, mais de manière extrêmement cloisonnée, comme par exemple des missions religieuses, des organisations non gouvernementales internationales... 
communautés dans ce processus contractuel dépend de la forme qu'elles revêtent et du mode de leur représentation : entité juridique formelle, individus désignés par la population... Dans tous les cas, les communautés prennent part aux contrats, soit directement avec les producteurs de soins et/ou les financeurs, soit par l'intermédiaire de la tutelle qui fixe au préalable les règles de la coordination entre les différents partenaires au niveau local. Dans le cas où la communauté dispose de la personnalité juridique, il est alors tout à fait possible que la convention passée avec un producteur de soins confère un rôle actif aux populations dans la gestion du service de santé au niveau local : la convention peut, par exemple, prévoir que les usagers soient représentés dans le comité de gestion de la santé du district ou d'un établissement hospitalier. Toutefois, dans le cas où la personnalité juridique n'est pas reconnue aux communautés, elles ne peuvent exercer de rôle véritablement décisionnel dans l'organisation de la prestation de services sanitaires, tout au plus peut-on imaginer un rôle consultatif, conféré à des personnalités locales qualifiées.

\section{IMPLICATIONS ET LIMITES DES APPROCHES NÉO-INSTITUTIONNELLES DES SYSTÈMES DE SANTÉ SUBSAHARIENS}

Les recommandations récentes de la Banque mondiale (1998) conduisent à dégager quatre grands axes de politique sanitaire : la catégorisation des interventions de santé, la diversification du secteur de santé, la mise en œuvre de mécanismes d'incitation à la performance du système sanitaire et la redéfinition du rôle de l'État dans le secteur de la santé. Ces différents axes de politique sanitaire correspondent assez étroitement aux résultats de la théorie des coûts de transaction (Coase, 1937; Williamson, 1975, 1985, 1998) qui associe aux interactions individuelles des formes d'organisation particulières qui permettent, en minimisant les coûts de la coordination, d'en maximiser l'efficience - la performance.

\section{Les axes stratégiques des réformes préconisées}

Le premier axe de la politique préconisée par la Banque mondiale consiste à catégoriser les actions de santé, pour laisser à la charge du secteur public les interventions qui sont efficaces en termes de coûts ; 
ce thème est récurrent depuis la proposition de la stratégie des soins de santé sélectifs (Walsh et Warren, 1977), et le Rapport sur le développement dans le monde consacré à la santé (Banque mondiale, 1993). Le second axe préconisé consiste à diversifier le secteur de la santé, tant sur le plan du financement que sur le plan organisationnel; il s'agit de permettre une coopération entre les producteurs et les financeurs publics et privés. Les politiques varient alors selon la situation initiale du secteur, à dominance publique ou privée. Cette diversification du secteur sanitaire s'accompagne alors de la mise en pratique de mécanismes de régulation hybrides, différents selon les pays. Dans les cas où le secteur sanitaire est fortement dominé par le public, la Banque mondiale estime que des systèmes de quasi-marchés ou de concurrence organisée permettent d'éviter la limitation directe des dépenses publiques consacrées à la santé. Redéfinir une nouvelle interface entre secteurs public et privé est préférable à la privatisation du secteur de la santél. En outre, lorsque les capacités institutionnelles des pays sont limitées, la Banque préconise une approche graduelle, qui met l'accent sur la décentralisation des services de santé et la mise en œuvre de marchés internes au secteur public, avant de mettre en place la coopération avec le privé. Le troisième volet de la politique de santé consiste à mettre en place des mécanismes incitatifs à la performance du secteur : les acteurs publics et privés doivent être encouragés à rechercher des solutions pour améliorer l'accessibilité des services de santé, ainsi qu'à procéder à une évaluation épidémiologique et économique afin de diffuser l'information sur les ratios coût-efficacité des interventions de santé. Par ailleurs, la mise en place de mécanismes de paiement permettant une meilleure maîtrise des coûts et l'engagement des professionnels de santé dans l'amélioration de la gestion de la santé est nécessaire. Les bailleurs de fonds internationaux, comme la Banque mondiale, doivent alors travailler non plus seulement avec les ministères de la Santé des pays bénéficiaires, mais également avec les ministères des Finances, de la Privatisation et du Plan. Enfin, les gouvernements doivent jouer un rôle plus important dans le financement des services de santé par le biais des fonctions de réglementation, de soustraitance et de subventions directes. Par ailleurs, la Banque mondiale préconise d'assurer un financement soutenable pour les dépenses récurrentes, plutôt que d'assurer les dépenses en capital. Dans les pays les plus démunis, des efforts sont nécessaires pour assurer un cofinancement de la santé, entre l'État et les communautés.

1. Rebalancing the public-private interface will be preferable to an outright privatization of social assets (World Bank, 1998, p. 18). 
Ainsi le mode d'intervention de la puissance publique dans les pays en développement est-il reconsidéré, entre autres, par la Banque mondiale, dans un sens qui n'est pas sans rappeler la théorie de Williamson. Cette dernière permet en effet de relier la nature d'une intervention spécifique de santé à l'arrangement institutionnel - le mode de gouvernance - efficient pour réguler sa production, tout en prenant en considération le contexte de la régulation. Appliquée au secteur de la santé, la théorie néo-institutionnaliste, et plus particulièrement la théorie des coûts de transaction, vise à déterminer les formes organisationnelles qui assurent la plus grande efficience au dit secteur. Les travaux qui, dans cette perspective, cherchent à hybrider les régulations marchandes et étatiques du secteur de la santé sous-tendent par ailleurs la mise en œuvre du managed care dans les pays développés'. Dans les pays en développement, un des objectifs affiché explicitement par les recommandations internationales (OMS, 2000) consiste à améliorer les "performances " des systèmes de santé. Cette performance, assimilable à la recherche de l'efficience sous contrainte d'équité, passe alors par une coopération accrue de l'ensemble des acteurs du système, sans distinction de statuts juridiques. Cette coopération au sein d'arrangements institutionnels ou de modes de gouvernance permet alors de réguler à la fois la production et le financement des activités de santé : la forme adéquate de ces modes de gouvernance est conditionnée par la nature des interventions de santé considérées, mais aussi par les caractéristiques de la relation qui se noue entre les différents acteurs du système de santé - la transaction, selon Williamson.

\section{Les attributs des transactions déterminent les modes de gouvernance du secteur de la santé}

Les transactions, définies par des contrats formels ou non, possèdent deux dimensions : leur fréquence et la spécificité des actifs, supports de la transaction. Outre ces caractéristiques, les transactions sont soumises à un degré plus ou moins important d'incertitude, tant en ce qui concerne la nature du produit échangé - sa qualité - que les comportements des co-échangistes. Ces différentes caractéristiques permettent alors d'associer aux transactions un coût qu'il s'agira de minimi-

1. Dans un rapport de 1998 , le Centre de recherches d'études et de documentation en économie de la santé (CREDES) insiste sur la diffusion de modèles de mise en concurrence des divers acteurs des systèmes de santé, concurrence sous-tendue, en théorie, par des travaux néo-institutionnalistes (Bocognano et al., 1998). 
ser afin d'améliorer l'efficience de chaque transaction. Ainsi, à chaque type de transaction va correspondre un mode de contractualisation - governance structure - différent.

- La fréquence des transactions détermine la forme juridique du contrat qui les définit. Williamson (1985), après McNeil (1978), distingue trois catégories de contrats formant la base juridique des modes de gouvernance possibles. Le contrat classique, tout d'abord, qualifie un accord dans lequel les parties en présence et le contenu du contrat - c'est-à-dire le produit échangé - sont clairement identifiés. Il n'est pas besoin de structures particulières pour gérer l'accord, et ce type de contrat fonctionne d'autant mieux que la transaction qu'il organise est ponctuelle. La vente - achat - de médicaments, par exemple, illustre particulièrement bien ce type de contrat. Le contrat néo-classique, ensuite, est une forme d'accord qui implique, outre les co-échangistes, une tierce partie dont l'activité consiste à arbitrer la transaction en cas de litige. Le contrat néo-classique fonctionne d'autant mieux qu'il concerne des relations qui ne sont plus ponctuelles et que l'incertitude, quant aux aléas qui peuvent surgir dans cette relation, est importante. Les accords de sous-traitance de certaines activités par un hôpital sont un exemple de contrat néo-classique, dans la mesure où le recours à l'autorité de tutelle ou aux tribunaux peut être requis en cas de manquement d'une des parties à ses obligations. Enfin, lorsque la relation est véritablement durable entre les individus, et que l'incertitude est forte, un troisième type de contrat, le contrat évolutif, est le plus efficient. Il s'agit, dans ce cas, d'un ensemble de règles qui encadrent l'accord et qui confèrent à l'un des cocontractants le soin de les interpréter en cas de litige. Dans le domaine de la santé, la concession d'un service de santé à un partenaire privé par un gouvernement qui en conserve la tutelle relève typiquement du domaine des contrats évolutifs. Cette dimension juridique se combine en outre à une dimension économique de spécificité des actifs. Un actif est dit spécifique s'il n'est redéployable dans une autre utilisation qu'à un coût prohibitif. Selon Williamson (1985), la spécificité des actifs se réfère aux investissements durables effectués lors d'une transaction spécifique. Dans la mesure où les actifs ne sont pas redéployables, leurs propriétaires ont intérêt à poursuivre la relation qu'ils ont nouée avec leurs partenaires : ce type d'investissement est alors risqué, dans la mesure où il occasionne une perte de la valeur du capital engagé lors de la rupture, toujours possible, du contrat. En matière d'organisation des systèmes de santé, le degré de spécificité des actifs est important: les connaissances techniques d'un médecin sont difficilement redéployables dans une autre activité que la médecine, l'attachement d'une clientèle pour le même 
praticien possède aussi les caractéristiques d'un actif spécifique : elle est difficilement redéployable à faibles coûts. L'appartenance d'un personnel de santé à une communauté ou à une ethnie donnée peut également être considérée comme un actif spécifique...

- Le croisement de la fréquence des transactions avec la spécificité des actifs conduit à considérer deux structures polaires de gouvernance: le marché, adapté aux transactions peu fréquentes sur des actifs non spécifiques, et la hiérarchie - ou gouvernance unifiée adaptée, quant à elle, aux transactions idiosyncratiques et récurrentes. Dans ce dernier cas, le système de santé sera organisé sur le mode du monopole public. Ces deux types de structures de gouvernance constituent deux cas limites; entre eux il existe des "formes hybrides" parmi lesquelles la "gouvernance trilatérale " - qui renvoie aux transactions occasionnelles qui s'appuient sur des actifs moyennement spécifiques, et la "gouvernance bilatérale" - qui concerne les transactions récurrentes sur des actifs moyennement spécifiques. L'approche contractuelle des systèmes de santé s'inscrit précisément dans le cadre de ces formes hybrides de gouvernance.

La situation actuelle des systèmes de santé subsahariens, caractérisée par la diversité croissante des acteurs et la pluralité des fonctions qui leur sont attribuées, met en exergue la nécessité de se référer au cadre transactionnel de Williamson. L'approche contractuelle instrumentalise le besoin, pour les décideurs de la santé, de faire évoluer les transactions qui ont lieu au sein du système sanitaire, et les contrats qui leur sont associés, de la coordination par le marché ou la hiérarchie vers une coordination hybride, dont les formes contractuelles relèvent, selon la nature du bien ou du service considéré, du contrat néo-classique ou du contrat évolutif. Cette évolution institutionnelle des systèmes de santé permettra d'améliorer leur performances, sans toutefois en obérer l'équité.

Cependant, l'approche transactionnelle repose sur certaines hypothèses fondamentales qui sont extrêmement fortes et dont la validité et l'adaptation au contexte sanitaire sont critiquables. Notre critique des recommandations contractuelles se fonde alors sur celle de ses hypothèses.

\section{Une critique des hypothèses fondamentales de la théorie des coûts de transaction}

La théorie des coûts de transaction, si elle rénove dans une certaine mesure le paradigme néo-classique standard, reste sous-tendue par 
trois hypothèses fondamentales. La rationalité individuelle postulée devient une rationalité limitée (Simon, 1976), mais intéressée. Ce statut ambigu qui lui est conféré limite la portée des dispositifs incitatifs construits, dans le cadre des politiques de santé, pour améliorer la coordination des acteurs du secteur. Ensuite, la théorie des coûts de transaction adopte une perspective allocative de la coordination économique : le contexte des interactions individuelles est donné et figé, ce qui conduit les recommandations de politique à négliger ses possibles évolutions. Enfin, l'individualisme méthodologique sous-jacent à la théorie ne permet pas la prise en considération d'une dimension collective de l'organisation des systèmes de santé.

- Le statut ambigu de la rationalité limite la portée des dispositifs incitatifs proposés. Pour Williamson (1985), la recherche de l'intérêt personnel reste au cour des motivations de l'individu. Sa rationalité - c'est-à-dire sa capacité à adapter ses actions aux fins qu'il poursuit est cependant limitée - ou bornée. L'exercice de cette rationalité limitée est un facteur principal d'incertitude. En matière de santé, la rationalité individuelle du malade est forcément limitée : le patient ne connaît pas dans la majorité des cas les causes de sa maladie, et ne peut réagir "rationnellement", d'autant plus que certaines affections demeurent longtemps asymptomatiques. Néanmoins, en définissant l'opportunisme, Williamson adopte une conception de l'intérêt personnel plus forte que celle adoptée par la théorie standard, dans la mesure où l'opportunisme étend l'hypothèse du self interest pour prendre en considération la recherche de l'intérêt individuel par la ruse (Williamson, 1985).

Les politiques sanitaires s'appuient sur la présomption de rationalité limitée des individus, qu'ils soient producteurs ou consommateurs de soins. Dans la perspective williamsonnienne, la constitution des modes de gouvernance efficients suppose que les limites à la rationalité des individus les conduisent, pour une transaction donnée, à préférer se coordonner au sein d'organisations - formes hybrides ou hiérarchie - plutôt que par le marché, dès lors que le recours à ce dernier est plus coûteux. Cette hypothèse de rationalité limitée des individus fonde la typologie de la structure institutionnelle des systèmes de santé. Cela suppose en fin de compte que les individus puissent calculer $e x$ ante les coûts des transactions associés aux différentes possibilités de coordination, ce qui contredit avec l'hypothèse même de rationalité limitée. Dans le même ordre d'idées, les réformes sont mises en place par les autorités publiques; or, si les individus se trouvent dans l'incapacité d'évaluer les coûts de transaction ex ante, il en est évidemment de même pour les membres du gouvernement : comment, dans ce 
cas, préconiser tel arrangement institutionnel - mode de gouvernance - plutôt qu'un autre, dans la mesure où il n'est pas possible, avant sa mise en œuvre, d'en calculer le coût d'opportunité ?

Cette critique induit la suspicion à l'égard de l'efficacité des politiques recommandées : les réformes préconisées sont dans cette perspective sous-tendues par la mise en ouvre d'incitations à la recherche de l'efficience, au travers de mécanismes de rémunération des praticiens, de sanctions envers les décideurs publics qui dépassent le budget alloué primitivement ${ }^{1} \ldots$ Ces mécanismes permettent non seulement de lutter contre l'opportunisme potentiel des individus qui peuvent chercher à manipuler les règles de la coordination à leur profit, mais également de pallier les inconvénients de la rationalité limitée, dans la mesure où des procédures de surveillance accompagnent ces incitations. Cela suppose que les acteurs du système de santé n'agissent - ou ne réagissent - qu'en fonction de calculs de maximisation de leur propre intérêt. En matière de santé, les actions individuelles sont-elles exclusivement coordonnées par le biais de l'intérêt personnel? Quid des relations de confiance entre les patients et leur médecin - que ce dernier soit un guérisseur traditionnel ou un médecin moderne ne change finalement rien à l'affaire - ?

Évacuer la confiance de l'analyse pose certains problèmes dans la spécification des transactions en matière sanitaire. La relation médecin-malade n'est pas uniquement une relation économique, elle fait intervenir des dimensions psychologique et sociale importantes pour comprendre pourquoi un patient ira consulter un médecin plutôt qu'un autre, à qualité technique et prix identiques. D'autres déterminants du recours aux soins interviennent en outre dès lors qu'on considère le cas de la médecine traditionnelle où les croyances et les habitudes culturelles jouent un rôle fondamental ; ces croyances et habitudes échappent de la même manière que la confiance à l'analyse transactionnelle.

À un autre niveau, on peut également douter de la viabilité des politiques incitatives, dans la mesure où elles sont entreprises par des décideurs publics, eux-mêmes dotés d'une rationalité limitée : les incitations risquent alors de manquer leur cible. Ces différents éléments rendent discutable l'élaboration de mécanismes incitatifs qui ne

1. L'approche sectorielle des systèmes de santé, développée sur les fondements de la théorie des modes de gouvernance, trouve une résonance au niveau microéconomique dans un certain nombre de travaux qui s'intéressent aux dispositifs concrets de contractualisation. Ces travaux utilisent la démarche de la théorie de l'agence (Jensen, Meckling 1976; Charreaux, 1985) afin d'analyser et de déterminer la nature des incitations et des procédures de contrôle des transactions à mettre en ouvre (Vining, Globermen, 1999 ; Mills, 1998 ; McPeake, Banda, 1994 ; Benett et al., 1997). 
prennent en considération qu'une dimension strictement pécuniaire des motivations individuelles.

- La perspective d'allocation de ressources adoptée conduit à négliger les dimensions contextuelles de la régulation sanitaire. L'analyse des modes de coordination proposée par Williamson reprend également le programme de recherche néo-classique dans son objectif de modéliser la répartition des ressources entre les acteurs de l'économie. Ce faisant, elle considère que la production des ressources n'est pas un problème spécifique de l'analyse économique, dans la mesure où produire équivaut à combiner des ressources préexistantes donnant naissance à un output, c'est-à-dire à une marchandise. Ainsi, le marché et les autres formes d'organisation de cette répartition des ressources constituent des modes de coordination économiques substituables, dans le sens où leur vocation est, en définitive, d'allouer des ressources.

En matière de santé, la conception de la régulation, défendue tant par les théoriciens néo-institutionnalistes que par la Banque mondiale, constitue une alternative organisationnelle au marché des soins; toutefois la forme hybride ne fait qu'assurer le rôle du marché - allouer des ressources - lorsque ce dernier est inefficace pour des raisons tenant à la fois aux caractéristiques des interventions de santé - services collectifs, services donnant naissance à des phénomènes d'externalité... - et aux caractéristiques du contexte dans lequel elles ont lieu - communautés rurales, urbaines, poids des croyances, degré de diffusion des infrastructures sanitaires, structures politique, juridique et sociale... Les interventions sanitaires comme les soins de santé primaires, qui impliquent à la fois les pouvoirs publics locaux, les communautés et les producteurs de soins publics et privés - voire traditionnels -, seront gérés par des modes de gouvernance hybrides au sens de Williamson (1985). Ces formes hybrides se positionnent comme autant de modes d'allocation des ressources se distinguant à la fois du marché et de la gestion publique monopoliste du secteur de la santé.

Dans cette perspective d'allocation de ressources, le contexte des décisions individuelles, c'est-à-dire celui de la contractualisation, est exogène : il contribue à déterminer les modes de gouvernance efficients pour gérer le système de santé. La formation de ce contexte est alors évacuée de l'analyse. Or il semble bien que le changement de contexte des choix organisationnels soit une dimension importante de la gouvernance efficiente des interventions et des systèmes de santé dans leur ensemble. Ainsi, selon la Banque mondiale (1998), les politiques mises en œuvre permettent de faire changer les mentalités, en termes de recours aux soins, de bonne gestion des infrastructures, de discipline budgétaire des dirigeants, etc. 
La mise en œuvre des réformes implique qu'on étudie au préalable les processus de structuration historique des éléments contextuels de la régulation sanitaire. Cependant, dans une économie d'allocation de ressources, où les différents modes de coordination peuvent se substituer les uns aux autres, la prise en compte de l'histoire, des irréversibilités temporelles, des processus de formation des préférences individuelles, du pouvoir, de la confiance ne constitue pas l'objet de l'analyse. Il serait pourtant fructueux de se pencher sur ces éléments, dans la mesure où il semble intéressant de s'interroger sur l'intangibilité du degré de spécificité des actifs nécessités par les transactions en matière de santé ; la perspective d'une économie de création de ressources (Brousseau, 1993) permet en effet d'analyser l'émergence de la spécificité de certaines ressources, comme le savoir-faire en gestion des unités de santé ou la formation des croyances et des habitudes qui sous-tendent le recours aux producteurs de soins, dimensions fondamentales de la régulation sanitaire. Le contexte des choix individuels serait alors incorporé dans l'analyse.

- L'individualisme méthodologique nie tout caractère collectif à l'organisation du système de santé. Enfin, l'économie néo-institutionnelle, à l'instar de la théorie standard, relève de l'individualisme méthodologique, mais a toutefois comme particularité de déduire l'émergence de modes de gouvernance systémiques d'interactions individuelles - les transactions. La théorie des coûts de transaction depuis Coase (1937) montre l'organisation comme un mode de coordination des décisions individuelles différent du marché. Celui-ci existe parce qu'il permet de minimiser les coûts du recours au système de prix : les coûts de transaction. Ainsi, «l'objectif de la nouvelle économie institutionnelle était de démontrer l'efficience sociale des modes de coordination non conformes aux enseignements de la théorie de la concurrence pure et parfaite" (Brousseau, op. cit.).

Les expériences de privatisation des systèmes de santé illustrent particulièrement ce point : la coordination interindividuelle par les prix ne conduit pas à l'optimum, dans la mesure où un certain nombre de services de santé ne possèdent pas les attributs de biens privés, potentiellement répartis de manière optimale par le marché. Les actions de santé présentent en effet, pour certaines d'entre elles, des caractéristiques de biens collectifs, ou engendrent des externalités de consommation; en conséquence, selon Samuelson (1954), ces services doivent être produits et/ou financés par l'autorité publique.

Les modes de coordination sanitaires - ou modes de gouvernance - préconisés par la Banque mondiale et les autres organisations internationales chargées de l'aide aux secteurs de santé dans les pays 
les plus démunis mettent en exergue la nécessité de diversifier le secteur sanitaire. Dans cette perspective, ces acteurs publics doivent œuvrer, aux côtés des acteurs privés, à la poursuite des objectifs décrits dans la stratégie sectorielle de santé, en particulier celui qui vise à améliorer les performances - organisationnelles et financières - des systèmes de santé. Cette diversification du système sanitaire implique la mise en relation de secteurs - publics et privés - autrefois extrêmement cloisonnés. Les recommandations sous-entendent alors une formulation de ces relations par l'approche contractuelle.

La catégorisation des interventions de santé et le lien entre nature du service et mode de gouvernance montrent la filiation théorique " contractualiste" des recommandations de la Banque mondiale et de l'OMS. Les gouvernements doivent tenir compte de la spécificité des interventions sanitaires et déléguer les fonctions de production ou de financement qui étaient les leurs à d'autres acteurs - gouvernementaux ou non. Différents modes de délégation existent alors, de la sous-traitance à la concession de service public, qui tous reposent sur un contrat entre les différents acteurs. Par ailleurs, lorsque le but de la politique économique de santé consiste à réglementer le secteur privé de la santé, il s'agit en fait d'édicter des règles de bon fonctionnement $\mathrm{du}$ marché. Ces règles sont formalisées par des contrats passés entre l'autorité publique et les producteurs de soins privés, dont les clauses peuvent définir, par exemple, un standard de qualité.

En définitive, les arrangements institutionnels recommandés par les organisations internationales présentent la particularité de conceptualiser, à l'instar de Williamson, l'institution comme un "nœud de contrats » qui lie les divers intervenants du système de santé. Dans cette perspective, deux niveaux de régulation s'articulent : une régulation par intervention en matière de santé, d'une part ; une régulation systémique, d'autre part.

Au niveau d'une intervention en matière de santé, le contrat est passé entre les différents producteurs de soins, les financeurs et éventuellement les représentants de l'autorité publique. En outre, les consommateurs de soins sont susceptibles de se lier à leur tour avec des producteurs de soins - dans le cas de mécanismes de rémunération à l'épisode - ou avec des financeurs - adhésion à une assurance privée ou à une mutuelle locale, voire à un système d'assurance sociale lorsque cela est possible. Les relations entre les différents intervenants sont alors considérées comme autant de contrats bilatéraux mutuellement avantageux qui permettent d'assigner, à chaque intervention de santé, une structure de gouvernance efficiente. 
Au niveau du système de santé dans son ensemble, les recommandations des organisations internationales visent à réintroduire un rôle actif de l'État dans la santé. À côté des fonctions traditionnelles de production directe ou de financement, les autorités publiques possèdent en effet le devoir de fixer les règles générales de coordination entre les partenaires du système de santé ; la décentralisation des décisions en matière de politique sanitaire locale, les modalités d'ouverture du domaine d'intervention autrefois public au secteur privé en constituent des exemples fréquents. L'État se trouve alors en relation avec les autres producteurs de soins, ainsi que les différents bailleurs de fonds - organismes internationaux, organisations non gouvernementales... Ces relations sont contractuelles, au sens de la théorie des coûts de transaction, dans la mesure où elles posent le problème de l'économie du système de santé comme un problème de contrats passés entre les différentes parties prenantes des systèmes de soins, orienté vers l'objectif d'efficience du système de santé dans son ensemble.

Cependant, la recherche d'efficience constitue-t-elle vraiment le seul moteur de l'intervention de l'État dans la santé ? Quid alors du pouvoir, de la politique, des "instincts prébendiers » qui guident les décisions de certains chefs d'État? La question des jeux de pouvoirs se pose alors en des termes qui nous éloignent à la fois de la constitution d'arrangements institutionnels visant à la recherche de l'efficience, mais également de la perspective contractuelle de Williamson qui, par la même voie qu'il emprunte pour évincer la confiance, écarte le pouvoir de l'analyse. L'institution n'est pas seulement une institution économique; elle organise des relations sociales et juridiques, selon des modalités qui mettent en perspective, à côté de la recherche d'efficience, le rôle des habitudes, des coutumes, des valeurs morales et éthiques, qui sont étrangères à l'analyse individualiste des institutions.

De manière connexe, si le rôle de l'État se trouve évoqué, dans une perspective qui conduit à redéfinir son champ d'action dans le domaine de la santé, la logique de son fonctionnement interne et le rapport que ce dernier entretient avec les citoyens - expression de la souveraineté - sont rarement étudiés. Pourtant, la manière dont s'exerce la souveraineté n'est-elle pas sous-tendue par des valeurs morales qui guident à leur tour l'organisation d'un système de santé ? Plus particulièrement, la définition de l'équité, prise comme une valeur morale, n'influence-t-elle pas la nature et l'organisation d'un système de santé et de protection sociale? 
Dans un article paru en 2001, Bernard Hours constate que, après plusieurs décennies de socialisation de la santé, c'est un marché de la santé qui s'installe (Hours, 2001). Les recommandations des organisations internationales visent dès lors à permettre à ces " marchés » de la santé de mieux fonctionner dans les pays en développement. Les politiques proposées permettent en effet d'instaurer une régulation des systèmes sanitaires qui se rapproche du managed care en œuvre dans les pays industrialisés. La mondialisation de ce managed care contribue alors à considérer ce modèle comme un véritable nouveau paradigme du développement des systèmes de santé.

Plus précisément, les critiques adressées aux approches néoinstitutionnelles permettent de mettre en exergue les enjeux auxquels se trouvent confrontés les différents acteurs des systèmes de santé des pays en développement, au premier rang desquels les populations ellesmêmes.

La santé des populations est remise en question sur au moins deux plans: d'une part, la recherche de l'efficience de systèmes de santé sous-financés implique une catégorisation des interventions de santé extrêmement étroite. Cette restriction du domaine de la santé publique conduit par exemple à rejeter en dehors de ce champ les traitements antirétroviraux du VIH/SIDA, alors même que l'épidémie en Afrique subsaharienne ne fait que s'étendre. D'autre part, les dispositions contenues dans la réforme contractuelle des systèmes de santé reviennent à considérer la santé comme une marchandise (Hours, 2001) dont la consommation relève de la responsabilité de chacun. Or la plupart des problèmes de santé des populations africaines ressortissent du domaine d'une santé collective, intégrée dans un ensemble de coutumes et de valeurs qui sont écartées de l'analyse "rationaliste » des approches contractuelles.

La prise en charge équitable des malades est également en cause. La diversification des acteurs des systèmes de santé conduit à faire émerger une concurrence au sein du secteur sanitaire dans son ensemble : l'expansion d'un secteur privé à but lucratif - hors ONG impose une contrainte de rentabilité de sa clientèle. Dans le contexte africain de pauvreté généralisée, il est à craindre que seule la frange la plus aisée de la population puisse bénéficier de soins de santé de qualité, tandis que les plus démunis n'auraient accès qu'à un ensemble de 
plus en plus étriqué de services publics. C'est une conception de l'équité a minima qui émerge des recommandations internationales en matière de politiques de santé, tandis que c'est a maxima que la pauvreté et les mauvaises conditions sanitaires frappent les populations.

Enfin, et plus généralement, cette critique s'inscrit dans l'espace plus large de la mondialisation des politiques de santé et de développement (Lee, Goodman, 2002). L'internationalisation de la pensée néolibérale occidentale se traduit, dans les recommandations contractuelles de politique sanitaire, par une recherche d'efficacité économique ou technique des systèmes de santé publics, au détriment de considérations sociales, culturelles, morales ou plus généralement structurelles qui rendent pourtant caduques les hypothèses des modèles traditionnels. Tant la mixité public/privé du secteur de la santé que le managed care, en pratique dans la majeure partie des pays du monde, sont-ils vraiment à l'ordre du jour dans des régions où la faillite des institutions et du marché appellent directement la renégociation d'un contrat social? L'échec des plans d'ajustement structurel et de leurs conséquences sociales aurait pourtant dû alerter les « experts » des organisations internationales sur les dangers de la translation des modèles occidentaux aux pays en développement.

Ces approches, fondées au moins en partie sur les théories néoinstitutionnelles, proposent une vision individualiste, contractuelle et allocative de l'organisation des systèmes de santé. Un véritable « nouveau paradigme " du développement des systèmes de santé dans les pays pauvres se devrait alors d'intégrer, à côté des variables individuelles traditionnelles, des éléments structurels de l'ordre du collectif qui influent à un degré plus ou moins important sur les comportements en matière de coordination sanitaire. Cela permettrait certainement d'ouvrir de nouvelles pistes pour réformer les systèmes de santé.

BIBLIOGRAPHIE

Banque mondiale (1993), Rapport sur le développement dans le monde. Investir dans la santé, Banque mondiale, Washington.

Bennett S. et al. (1997), Public-private roles in the pharmaceutical sector: Implications for equitable access and rational drug use, Health Economics and Drugs, Geneva, WHO DAP, series No. 5.

Bocognano A. et al. (1998), Mise en concurrence des assurances dans le domaine de la santé. Théorie et bilan des expériences étrangères, Rapport CREDES, CREDES, Paris.

Brousseau E. (1988), De nouvelles approches pour l'entreprise, Revue d'économie industrielle, $\mathrm{n}^{\circ} 46$, p. 84-90. 
Carrin G, et al. (1997), L'approche contractuelle: de nouveaux partenariats pour la santé dans les pays en développement, Document technique de l'OMS, ref. : WHO/ICO/MESD, 24, Genève.

Charreaux G. (1985), La théorie positive de l'agence : une synthèse de la littérature, in G. Charreaux et al., De nouvelles théories pour gérer l'entreprise, Paris, Economica, p. 21-55.

Coase R. (1937), The Nature of the Firm, Paris, Economica, p. 386-405. Trad. franç. (1987), Revue française d'économie, vol. II, $\mathrm{n}^{\circ} 1$, p. 133-163.

Favereau O. (1989), Marchés internes, marchés externes, in J.-P. Dupuy et al., "L'économie des conventions », numéro spécial de la Revue économique, $n^{\circ} 40,2$ mars, p. 273-328.

Hours B. (2001), De l'offre de soins au prix de la santé et à la marchandise, Économies et sociétés, série F, n' 39, p. 1491-1504.

Hugon P. (1991), La pensée française en économie du développement, Revue d'économie politique, $\mathrm{n}^{\circ} 2$, p. 171-229.

Jacquemot P. (1988), La désétatisation en Afrique subsaharienne. Enjeux et perspectives, Revue Tiers Monde, t. XXIX, n 114, p. 271-293.

Jensen M. C. et Meckling W. C. (1976), Theory of the firm, manageral behaviour, agency costs and ownership, Journal of Financial Economics, vol. 3, $n^{\circ} 4$, p. 305-360.

Lee K., Goodman H. (2002), Global policy networks : The propagation of health care financing reform since the 1980 s, in K. Lee, K. Buse, S. Fastukian (2002), Health Policy in a Globaising World, Cambridge, Cambridge University Press.

McNeil I. R. (1978), Contracts : Adjustment of long term economic relations under classical, neoclassical and relational contracts law, Northwestern University Law Review, vol. 72, n 6, p. 854-905.

McPake B, Banda E. (1994), Contracting out of health services in developing countries, Health Policy and Planning, vol. 9, $\mathrm{n}^{\circ} 1$, p. 25-30.

Mills A. (1998), To contract or not to contract? Issues for low and middle income countries, Health Policy and Planning, vol. 13, $\mathrm{n}^{\circ} 1$, p. 32-40.

Musgrave R. A. (1959), The Theory of Public Finance, New York, McGrawHill.

Nolan B., Turbat V. (1995), Cost Recovery in Public Health Services in Subsaharan Africa, Washington, World Bank.

Parker D., Knippenberg R. (1991), Financement et participation communautaire: un examen de la question, Communication au séminaire du CIE, Paris.

Perrot J. (1999), L'approche contractuelle comme outil de mise auvre des politiques nationales de santé, Document de travail non publié, Genève, OMS.

Samuelson P. A. (1954), The pure theory of public expenditure, Review of Economics and Statistics, p. 387-389.

Tizio S. (1999), La coordination dans les économies en développement. Une contribution théorique à la régulation des systèmes de santé subsahariens, thèse de doctorat ès sciences économiques, Université de Bourgogne, Dijon. 
Tizio S., Flori Y. A. (1997), L'initiative de Bamako: "santé pour tous " ou "maladie pour chacun»?, Revue Tiers Monde, t. XXXVIII, n ${ }^{\circ}$ 152, p. $837-858$.

Walsh J., Warren K. (1979), Selective primary health care : An interim strategy for desease control in developing countries, New England Journal of Medicine, $\mathrm{n}^{\circ}$ 301, p. 976-984.

Williamson O. E. (1975), Market and hierarchies, New York, The Free Press.

Williamson O. E. (1985), The Economic Institutions of Capitalism, New York, The Free Press. Trad. franç. (1994), Les institutions de l'économie, Paris, InterÉditions, 1994.

Williamson O. E. (1998), The institutions of governance, American Economic Review, 88 (2), p. 75-79.

World Bank (1998), Health, Nutrition and Population, Sector strategy paper, Washington, World Bank. 